\title{
An assessment of the effect of black tea on glucose and insulin response in vivo to a standard carbohydrate-rich food
}

\author{
S. L. Hull ${ }^{1}$ and J. E. Brown ${ }^{2}$ \\ ${ }^{1}$ Nutrition Research Section, Leatherhead Food Research, Randalls Road, Leatherhead, Surrey, KT22 7RY, \\ UK and ${ }^{2}$ Division of Nutritional Sciences, Faculty of Health and Medical Sciences, University of Surrey, \\ Guildford GU2 7XH, UK
}

Beverages constitute a major component of Western diets, and tea is consumed widely in the UK and is linked to a variety of health benefits $^{(1)}$. Important tea components such as catechins stimulate insulin secretion and tea flavonoids can inhibit carbohydrate-digesting enzymes $^{(2)}$. Together these properties could delay carbohydrate digestion and absorption. Furthermore, there is evidence to suggest that tea polyphenols may inhibit salivary $\alpha$-amylase ${ }^{(3)}$, and therefore reduce the rate of carbohydrate digestion and absorption which may be important in terms of diabetes risk. We hypothesised that consuming black tea with a carbohydrate-rich food might reduce the glucose and insulin responses to that food.

Black tea (Tetley Group (UK) Ltd.) was used for the entire study. The composition of this tea was determined by LC-MS analysis ${ }^{(4)}$. Ethical approval was obtained from East Kent Research Ethics Committee and twelve healthy participants completed the study. Each participant consumed each test meal on two separate occasions after an overnight fast. The test beverages were: $200 \mathrm{ml}$ hot water (control); $200 \mathrm{ml}$ black tea infused for $0.5 \mathrm{~min}$; and $200 \mathrm{ml}$ black tea infused for $3 \mathrm{~min}$. Each beverage was consumed simultaneously with a portion of cornflakes containing $50 \mathrm{~g}$ available carbohydrate. Capillary blood samples were taken at regular intervals and the primary outcome measure was a change in the $30 \mathrm{~min}$ blood glucose measurement.

Although there were small differences between the profiles obtained for glucose and insulin, no significant differences in plasma glucose or insulin responses were observed between the three test meals. This study therefore demonstrated that black tea had no effect on the glucose and insulin responses to a starch rich food.

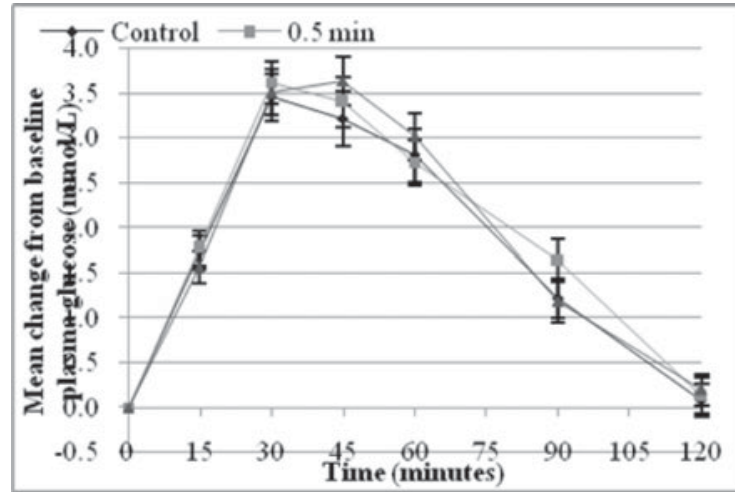

Fig. 1. Glucose response.



Fig. 2. Insulin response.

Several factors may account for this non-significant result. Although tea catechins have been shown to inhibit salivary $\alpha$-amylase activity, the amount of these catechins in the tea infusion may have been too small to elicit a significant effect. Flavonols and theaflavins may also inhibit salivary $\alpha$-amylase but there is evidence to suggest that caffeine may stimulate $\alpha$-amylase. It is possible that in this study the caffeine present in the tea stimulated $\alpha$-amylase activity while the tea polyphenols inhibited $\alpha$-amylase activity, resulting in no net change in glucose or insulin response. Further work should be carried out with decaffeinated tea to remove the confounding effects of caffeine.

1. Khan N \& Mukhtar H (2007) Life Sci 81, 519-533.

2. Bryans J, Judd P \& Ellis P (2007) J Am Coll Nutr 26, 471-477.

3. Lo Piparo E, Scheib H, Frei N et al. (2008) J Med Chem 51, 3555-3561.

4. Clifford MN, Knight S \& Kuhnert N (2005) J Agric Food Chem 53, 3821-3832. 